\title{
CrimRxiv
}

\section{Neuroforensics: Exploring the Legal Implications of Emerging Neurotechnologies}

National Research Council

Published on: Jul 06, 2018

License: Creative Commons Attribution 4.0 International License (CC-BY 4.0). 
